\title{
Preliminary Evaluation of Six Current Bilirubin Adsorbents on Potential Application for the Hemoperfusion of Severe Neonatal Jaundice
}

\author{
Kai_Wang Ma*, Shu_Ying Feng and Jian_Ying Yang \\ College of Medical technology and Engineering, Henan University of Science and Technology, Luoyang, 471003, PR \\ China
}

\begin{abstract}
Background: Now hemoperfusion has not been used for the treatment of severe neonatal jaundice, and bilirubin adsorbent with high adsorption and safety should be the prerequisite for the use.

Objectives: To investigate the adsorption of six kinds of current adult/industrial bilirubin adsorbents from severe neonatal jaundice plasma, then screen the best one with highest adsorption for further evaluations, with an aim to provide preliminary experimental reference for the potential clinical use of hemoperfusion in newborn.
\end{abstract}

Methods: Six kinds of current bilirubin adsorbents [NKA-9, NK-110, polymethacrylic acid (PMMA), polyvinyl alcohol (PVA), chitosan and burning resin] were selected for the investigation. Under the proportion of 1 portion of adsorbent ( $\mathrm{g}$ ) to 2 portion of severe neonatal jaundice plasma $(\mathrm{ml})$, the adsorbent was allowed to dynamically contact the plasma (recycled from the discarded plasma of severe neonatal jaundice inpatients after exchange transfusion treatment) at $37{ }^{\circ} \mathrm{C}$ for $1 \mathrm{~h}$ respectively, then their adsorption for bilirubin was calculated and compared, and then based on the comparative result, the adsorbent with best adsorption was subject to further evaluation such as its effect on the blood elements, the hemolysis system and blood clotting system of newborn.

Results: Under the same test conditions, the adsorption of NKA-9 for bilirubin was the best and up to $85 \%$, and its effect on other physiological indexes was clinically acceptable.

Conclusion: The preliminary evaluations offer us the knowledge and reference of hemoperfusion therapy being potentially applied in the treatment of severe neonatal jaundice, as well as other shortcomings existing within the therapy to be overcome.

Keywords: Bilirubin, adsorption, neonatal jaundice, newborn, hemoperfusion.

\section{INTRODUCTION}

For the newborns, neonatal jaundice is the common disease [1]. There are two types of neonatal jaundice: one is physiological and the other is pathological. As for the physiological, it can be automatically recovered without any treatments. Whereas, for the pathological, it falls into three types: mild, moderate and severe. If they were not to be properly or promptly handled (esp. the severe one), it can cause kernicterus, and consequently sequela or even death may occur [2]. The pathogeny of neonatal jaundice [2] is that the amount of bilirubin overproduces so that it is beyond the excretive and metabolic capability of the neonatal body, thus results in the accumulation of redundant bilirubin. Therefore, the treatment should be based on how to reduce the level of bilirubin effectively and quickly. Now, three clinical therapies [3-5] (drug, blue phototherapy and exchange transfusion) are in use for the treatment. Drug can accelerate the excretive and metabolic process of bilirubin; however, it is now seldom used in clinic due to its great side effect [6]. Blue phototherapy can change the excretive and metabolic pathway of bilirubin, which is only suitable for mild sick neonatal but also has side effect [7-8]. Exchange transfusion

*Address correspondence to this author at the College of Medical technology and Engineering, Henan University of Science and Technology, Luoyang, 471003, PR China; E-mail: mkw007@126.com is now the last resort for the treatment of severe neonatal jaundice, in which there are some disadvantages, such as blood cross-infection, scarcity of blood source and operation complexity [9-10]. Because the incidence of severe neonatal jaundice is in rise $[1,11]$ and there exist some larvaceous disadvantages to be overcome for the exchange transfusion, in view of high safety, there is an imminent demand to develop a new, safer, more economic and simple treatment for severe neonatal jaundice to substitute exchange transfusion.

Hemoperfusion [4,12-13] has been clinically used since 1970s, whose characteristics are to clear pathogenic material directly and quickly from blood or plasma so as to purify the blood, and whose key depends on the perfusion adsorbent with high specificity and adsorption as well as good hemocompatibility [14-16]. Therefore, hemoperfusion has the potentiality to substitute exchange transfusion for the treatment of severe neonatal jaundice. Because of the newborn physiological particularity, the key to apply the hemoperfusion for the treatment depends on whether the bilirubin adsorbent is of high specificity, high adsorption and good hemocompatibility for the newborn blood. Now, several bilirubin adsorbents have been reported, such as NKA-9 [17], NK-110 [17], PVA [17], PMMA [18], chitosan [19] and burning resin [20], which are mainly used in industry to extract bilirubin or in clinic for adult 
hyperbilirubinemia hemoperfusion [21]. But within our knowledge, there is no report about their use in the hemoperfusion for severe neonatal jaundice and on their adsorption for bilirubin from severe neonatal jaundice plasma/blood. This study was aimed to test their adsorption properties upon neonatal jaundice plasma, and then screen out the best for further evaluation, with an aim to provide clinical experimental reference for the choice or development of bilirubin adsorbent for the hemoperfusion in severe neonatal jaundice.

\section{MATERIALS AND METHODS}

\subsection{Main Materials and Equipments}

Blood/plasma was obtained from newborn in patients with severe jaundice in the Chongqing Children's Hospital, China, and the blood/plasma were those that were recycled from the discarded blood/plasma of severe neonatal jaundice inpatients after exchange transfusion treatment; three bilirubin adsorbent candidates (NKA-9, NK-110 and PMMA) were provided from chemical plant of Nan_Kai University China; three bilirubin adsorbent candidates (PVA, chitosan and charring resin) were provided by Tianjin Jinlin Biochemical Development Company China. Coagulometer (CA1500, USA), Electrolyte Analyzer (XD685, USA), Automatic Biochemical Analyzer (Dimension RXL, USA), Blood Gas Analyzer (Bayer-860, USA), Specific Protein Detector (Nephster, Germany), Hemoperfusion Device (selfmade). (Note: NKA-9 and NK-110 are a kind of macroporous resin).

\subsection{Adsorption of the Candidates from Severe Neonatal Jaundice Plasma}

The adsorbent (15 g) was filled into the hemoperfusion column. The initial value of bilirubin level in the severe neonatal jaundice plasma was firstly assayed, then $30 \mathrm{ml}$ the plasma was allowed to circulate through the hemoperfusion column for $1 \mathrm{~h}$ at $37^{\circ} \mathrm{C}$. Finally the end value of bilirubin level was assayed after the circulations. With the same procedure and conditions, the rest were subjected to the same treatment respectively, and the one with best adsorption was screened out for further evaluation.

\subsection{Further Evaluations of NKA-9}

From section 2.2, NKA-9 did the best among the six; therefore, it was screened out for further evaluation.

\subsubsection{The Effects of NKA-9 on the Plasma Components of Severe Neonatal Jaundice}

$7 \mathrm{~g}$ NKA-9 was filled in the hemoperfusion column, then $14 \mathrm{~mL}$ severe neonatal jaundice plasma was allowed to circulate through the column at $37^{\circ} \mathrm{C}$ for $1 \mathrm{~h}$. Those biochemical indicators, such as plasma protein, indicators of renal function, inorganic ions, immune system, enzyme system and auxiliary system, were assayed before and after the circulation to investigate its effects on the blood elements and components.

\subsubsection{Effects of NKA-9 on the Coagulation System of Severe Jaundice Neonatal}

$7 \mathrm{~g}$ of NKA-9 was filled in the hemoperfusion column, then $14 \mathrm{~mL}$ severe neonatal jaundice plasma anticoagulated with 1:9 sodium citrate anticoagulant was allowed to circulate through the column at $37^{\circ} \mathrm{C}$ for $1 \mathrm{~h}$. Those indicators of coagulation system, such as prothrombin time (PT), activated partial thromboplastin time (APTT), fibrinogen (FIB) and prothrombin time (TT) were assayed before and after the circulation with CA1500. Similarly, $7 \mathrm{~g}$ NKA-9 was filled in the hemoperfusion column, then $14 \mathrm{~mL}$ severe neonatal jaundice plasma anticoagulated with heparin anticoagulant was allowed to circulate through the column at $37^{\circ} \mathrm{C}$ for $1 \mathrm{~h}$, then Complement indicator (C3) was assayed before and after the circulation with CA1500.

\subsubsection{Effects of NKA-9 on the Hemolytic System of Severe Jaundice Neonatal}

$7 \mathrm{~g}$ NKA-9 was filled in the hemoperfusion column; $10 \mathrm{~mL}$ out of $120 \mathrm{~mL}$ severe neonatal jaundice blood was taken for blank test, and $110 \mathrm{~mL}$ of the rest was divided into halves equally to separate plasma and blood cells respectively. The blood cells just isolated were temporarily preserved respectively. One portion of the plasma was allowed to circulate through the column at $37^{\circ} \mathrm{C}$ for $1 \mathrm{~h}$, whereas the other without circulation was as the control. After the circulation, the two portions of plasma were mixed with the original corresponding blood cells isolated respectively, and then the hemolytic indicators of the original, the treated and the untreated blood were measured respectively, such as red blood cells, hemoglobin, the average red cell volume, platelet count, WBC, the average amount of hemoglobin, hematocrit, mean hemoglobin concentration, erythrocyte distribution width, mean platelet volume, the absolute value of lymphocytes, neutrophils absolute, absolute value of intermediate cells, percentage of lymphocytes and neutrophilic leukocyte.

\section{RESULTS}

\subsection{Adsorption of Six Bilirubin Adsorbent Candidates from Severe Neonatal Jaundice Plasma}

Under the experimental conditions, their adsorptive capability was shown in Fig. (1). We can see NKA-9, PVA and burning resin had good adsorption for direct bilirubin (DB), among them, PVA ranked the top. NKA-9, PMMA resin and PVA had good adsorption for total bilirubin (TB), among them, NKA-9 ranked the top. Taken together, we considered the NKA-9 had the best adsorption for bilirubin from severe neonatal jaundice plasma.

\subsection{Effects of NKA-9 on the Components of Severe Neonatal Jaundice Plasma}

Under the experimental conditions, Table 1 listed the effect of NKA-9 on the components of severe neonatal jaundice plasma. According to the statistical analysis result, it showed that NKA-9 had insignificant effect on plasma biochemical parameters and also in line with basic clinical reference value.

\subsection{Effects of NKA-9 on the Coagulation System of Severe Neonatal Jaundice Blood}

Under the experimental conditions, Table 2 listed the effect of NKA-9 on the coagulation system of severe neonatal jaundice blood. According to statistical analysis, it showed that NKA-9 had insignificant effect on coagulation system indicators and also in line with basic clinical reference value. 
Table 1. The effects of NKA-9 on the Biochemical Parameters of Severe Neonatal Jaundice Plasma from 6 Cases

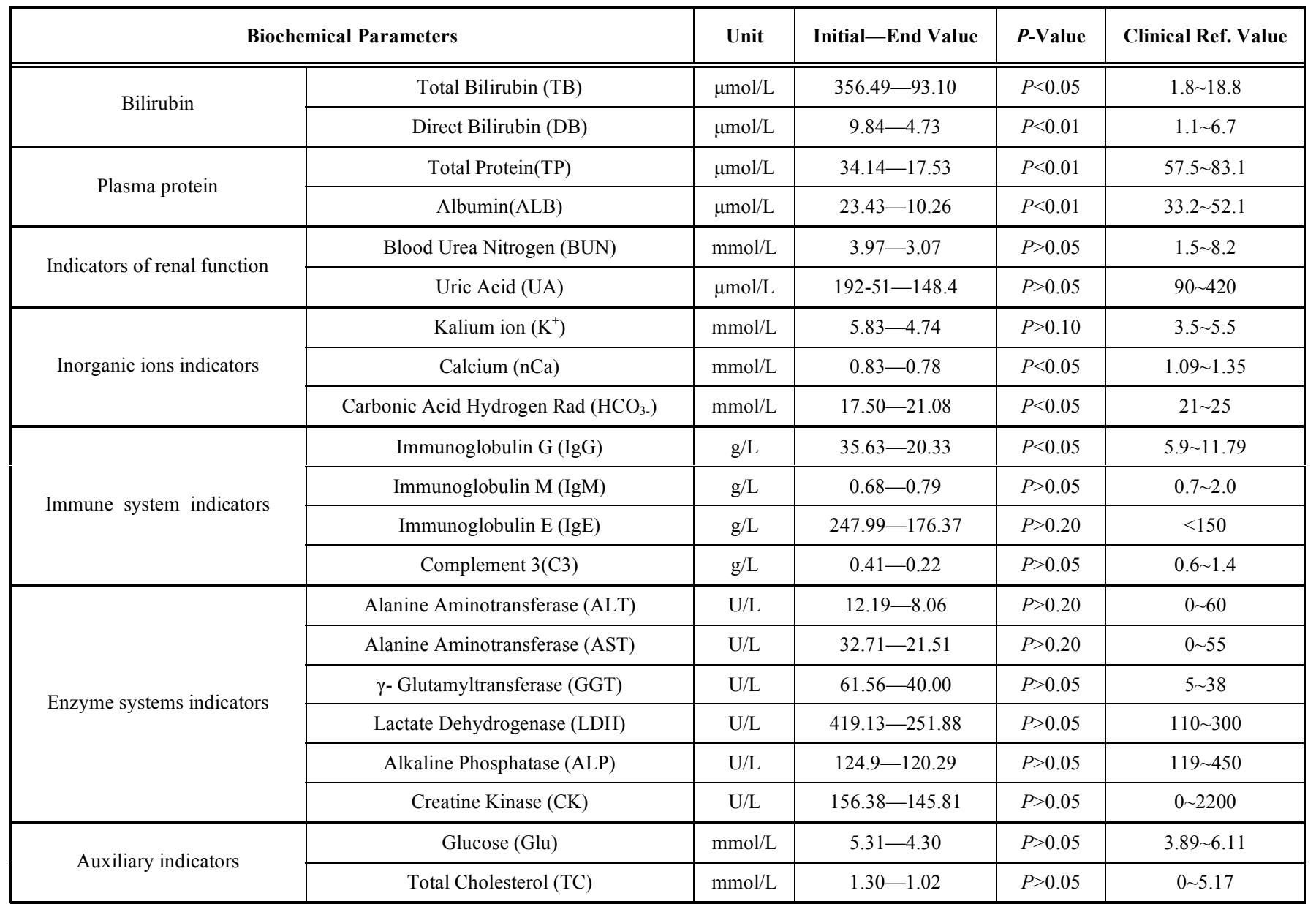

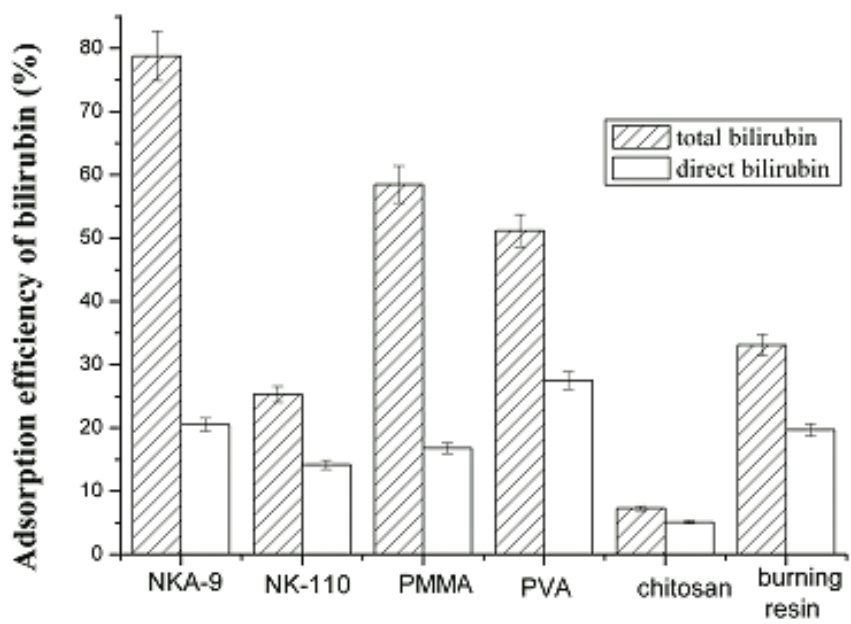

Fig. (1). Adsorption of six adsorbent candidates for bilirubin from severe neonatal jaundice plasma. Adsorbent $(\mathrm{g})$ : plasma $(\mathrm{ml})=1: 2$, $37^{\circ} \mathrm{C}$, hemoperfusion circulation time $=1 \mathrm{~h}, 3$ repeats records. Plasma from 20 cases.

\subsection{Effects of NKA-9 on the Hemolytic System of Severe Neonatal Jaundice Blood}

Under the experimental conditions, Table 3 listed the effect of NKA-9 on the hemolytic system indicators of severe neonatal jaundice blood. According to statistical analysis, it showed that NKA-9 had insignificant effect on hemolytic system indicators and also in line with basic clinical reference value.

\section{DISCUSSIONS}

According to the structure of bilirubin, the materials [1720] currently reported to have better adsorption for bilirubin are mainly NKA-9, NK-110, PMMA, PVA, chitosan and burning resin. NKA-9 is a kind of macroporous resin with cyano, which has been mainly used in industry to extract bilirubin due to its good affinity to bilirubin; NK-110 is a kind of macroporous resin, which has been reported for the treatment of adult hyperbilirubinemia perfusion due to its good affinity to macromolecules toxic substances; PMMA also has adsorption for bilirubin, as well as good biocompatibility and blood compatibility, which has been widely used in biomedical materials field; PVA has been widely used in enzyme immobilization and blood purification fields in the form of membrane or water gel; Polysaccharide is a newly-developing adsorption and separation materials; Burning resin is an adsorption material from a variety of polymer resin produced by hightemperature carbonization.

This preliminary evaluation indicated the level of TB and DB in the severe neonatal jaundice plasma was significantly reduced after the plasma was hemoperfused by the six kinds of bilirubin adsorbing materials respectively, $\mathrm{DB}$ is a more 
Table 2. The effect of NKA-9 on the Coagulation System Indicators of Severe Neonatal Jaundice Blood from 6 Cases

\begin{tabular}{|c|c|c|c|c|}
\hline Biochemical Parameters of Blood Clotting System & Unit & Initial一End Value & $\boldsymbol{P}$-Value & Clinical Ref. Value \\
\hline \hline Pro-time (PT) & $\mathrm{s}$ & $17.85 \sim 40.22$ & $P>0.20$ & $11 \sim 14.5$ \\
\hline Activated Partial Thromboplastin Time (APTT) & $\mathrm{s}$ & $72.03 \sim 88.70$ & $P>0.20$ & $26.5 \sim 41.5$ \\
\hline Fibrinogen (Fib) & $\mathrm{g} / \mathrm{L}$ & $1.83 \sim 0.83$ & $P>0.05$ & $2.0 \sim 4.0$ \\
\hline Thormbin Time (TT) & $\mathrm{s}$ & $25.78 \sim 33.65$ & $P<0.05$ & $14.0 \sim 21.0$ \\
\hline
\end{tabular}

Table 3. The Effects of NKA-9 on the Hemolytic System Indicators of Severe Neonatal Jaundice Blood from 8 Cases

\begin{tabular}{|c|c|c|c|c|c|c|}
\hline Medical Indicators & $\begin{array}{c}\text { Original } \\
\text { Blood } \\
\text { Sample }\end{array}$ & $\begin{array}{c}\text { Original Plasma } \\
\text { Mixed with Original } \\
\text { Erythrocyte }\end{array}$ & $\begin{array}{c}\text { Hemoperfused } \\
\text { Plasma Mixed with } \\
\text { Original Erythrocyte }\end{array}$ & $\begin{array}{l}\text { Clinic } \\
\text { ref. } \\
\text { Value }\end{array}$ & Unit & $\begin{array}{r}\text { Meeting } \\
\text { Clinical } \\
\text { Standard }\end{array}$ \\
\hline Hemoglobin & 120 & 138 & 140 & $110 \sim 160$ & $\mathrm{~g} / \mathrm{L}$ & $\sqrt{ }$ \\
\hline Mean Corpuscular Volume & 93.3 & 94.4 & 95.1 & $80 \sim 98.1$ & $\mathrm{fL}$ & $\sqrt{ }$ \\
\hline White Blood Corpuscle & 3.82 & 3.45 & 3.41 & $4.0 \sim 10.0$ & $* 10 \sim 12 / \mathrm{L}$ & $x$ \\
\hline Average Quantity of Haemoglobin & 33.3 & 33.1 & 33.1 & $27 \sim 34$ & $\mathrm{pg}$ & $\sqrt{ }$ \\
\hline Volume Packed Cells & 43.8 & 39.5 & 39.8 & $37 \sim 50$ & $\%$ & $\sqrt{ }$ \\
\hline Mean Hemoglobin Concentration & 340 & 337 & 336 & $320 \sim 360$ & $\mathrm{~g} / \mathrm{L}$ & $\sqrt{ }$ \\
\hline Modulus of Neutrophilic Granulocyte & 0 & 0 & 0.02 & - & $* 10 \sim 12 / \mathrm{L}$ & $\sqrt{ }$ \\
\hline Modulus of Ntermediate Cell & 4.3 & 4.7 & 3.4 & - & $* 10 \sim 12 / \mathrm{L}$ & $\sqrt{ }$ \\
\hline Pet of Leukomonocyte & 0.34 & 0.33 & 0.35 & $0.3 \sim 0.6$ & $\%$ & $\sqrt{ }$ \\
\hline Pet of Neutrophilic Granulocyte & 0.31 & 0.32 & 0.29 & $0.33 \sim 0.79$ & $\%$ & $x$ \\
\hline
\end{tabular}

important medical indicator for it reflects the function of liver. Among them, the adsorption of NKA-9 and PVA was the most significant; the adsorption ratio of NKA-9 was over $75 \%$, and the adsorption ratio of PVA was over $50 \%$. Although the level of $\mathrm{TB}$ and $\mathrm{DB}$ did not reduce to the normal level, the level had been below the threshold value of pathological neonatal jaundice $(204 \sim 255 \mu \mathrm{mol} / \mathrm{L})$, which can help the doctors and the patients buy time to create favorable conditions for other treatments of neonatal jaundice. Regarding to the aspect of blood compatibility, NKA-9 resin did not cause significant effects on most of blood components indicators of newborn, and those indicators were also in line with the clinical reference value [20-21]. Inevitably, some indicators had significant change. For example, the level of $\mathrm{HCO}_{3}$ - was below the normal value, which is adverse and unexpected in clinic treatment. However, such disadvantages can be easily remedied by other means. NKA-9 had major effects on the enzymatic system indicators of newborn, which is unexpected and need proper remedy. NKA-9 had little effects on the hemolytic system and blood clotting system indicators of newborn, which is favorable. Current studies [15,22] indicate hemoperfusion therapy for severe hepatitis is a good method for clearing bilirubin quickly. Due to hyperbilirubinemia becoming a common problem, therefore bilirubin adsorption therapy has great applicable prospect. In American, about $60 \%$ of 40 million neonatal may develop hyperbilirubinaemia [23], whereas $60 \%$ of hyperbilirubinaemia neonatal patients may develop into jaundice, of which pathological jaundice is higher than physiological one, whereas in China, incidence of pathological jaundice of newborn is up to $38.4 \%$ [24]. Because there still exist some drawbacks in current clinical therapies for pathological neonatal jaundice, improvements or new therapy is in urgent demand in order to gain great safety, therefore, how to cure neonatal jaundice (esp. the severe) with perfusion therapy and develop more effective, secure and economic bilirubin adsorbing material should be a novel challenge to the medical staff and scientists, and also has extensive market prospect. Unfortunately, the in vivo study cannot be now done because of the limitations of current technology.

\section{CONCLUSION}

The preliminary evaluations offer us the knowledge and reference of hemoperfusion being potentially applied in the treatment of severe neonatal jaundice; however, there still exists some problems to be resolved before it can be 
clinically used. Among six kinds of bilirubin adsorbing material candidates, NKA-9 resin had best adsorption for bilirubin from severe neonatal jaundice plasma, which may be expected as the adsorbing material to be used for the clinical hemoperfusion of neonatal jaundice disease with proper modification and therapeutic alliance. In future studies, people should develop new adsorbing material with high adsorption for bilirubin and with no/minor influences on other medical indicators except TB/DB, and/or seek compensative measurements to overcome the disadvantages causing by current bilirubin adsorbing materials.

\section{ACKNOWLEDGEMENTS}

This work was supported by the National Natural Science Fund (No.30900796) of China and the Doctor's research initial fund of Henan University of Science \& Technology China (No.09001284).

\section{REFERENCES}

[1] Mishra S, Agarwal R, Deorari AK, Paul VK. Jaundice in the newborns. Indian J Pediatr 2008; 75: 157-63.

[2] Boyd S. Treatment of physiological and pathological neonatal jaundice. Nurs Times 2004; 100: 40-3.

[3] Thomas JT, Muller P, Wilkinson C. Antenatal phenobarbital for reducing neonatal jaundice after red cell isoimmunization. Cochrane Database Syst Rev 2007; 2: CD005541.

[4] Chen HN, Lee ML, Tsao LY. Exchange transfusion using peripheral vessels is safe and effective in newborn infants. Pediatrics 2008; 122: e905-10.

[5] Boonyarittipong P, Kriangburapa W, Booranavanich K. Effectiveness of double-surface intensive phototherapy versus single-surface intensive phototherapy for neonatal hyperbilirubinemia. J Med Assoc Thai 2008; 91: 50-5.

[6] Murki S, Dutta S, Narang A, Sarkar U, Garewal G. A randomized, triple-blind, placebo-controlled trial of prophylactic oral phenobarbital to reduce the need for phototherapy in G6PDdeficient neonates. J Perinatol 2005; 25: 325-30.

[7] Raghavan K, Thomas E, Patole S, Muller R. Is phototherapy a risk factor for ileus in high-risk neonates. J Matern Fetal Neonatal Med 2005;18: 129-31.

[8] Sarin M, Dutta S, Narang A. Randomized controlled trial of compact fluorescent lamp versus standard phototherapy for the treatment of neonatal hyperbilirubinemia. Indian Pediatr 2006; 43:583-90.
[9] Sanpavat S. Exchange transfusion and its morbidity in ten-year period at King Chulalongkorn Hospital. J Med Assoc Thai 2005; 88: 588-92.

[10] Burkhard Z, Fredrik B. Conical intersection in a bilirubin model A possible pathway for phototherapy of neonatal jaundice. Chem Phys Lett 2006; 420: 556-61.

[11] Paulose NS, Hart D, Rauch D. Neonatal hyperbilirubinemia: an unexpected cause. Clin Pediatr (Phila) 2008; 47:388-94.

[12] Utsumi K, Kawabe M, Hirama A, et al. Effects of selective LDL apheresis on plasma concentrations of ICAM-1, VCAM-1 and Pselectin in diabetic patients with arteriosclerosis obliterans and receiving maintenance hemodialysis. Clin Chim Acta 2007; 377: 198-200.

[13] Zheng-Wei M, Li-Dong L, Kun L, Yu-Jun Z, Jia-Hong D, Improvement of graft function and animal survival by fat emulsion in liver transplant rats. Colloids Surf B: Biointerfaces 2007; 54(1): 25-32.

[14] Pan CJ, Tang JJ, Shao ZY, Wang J, Huang N. Improved blood compatibility of rapamycin-eluting stent by incorporating curcumin. Colloids Surf B: Biointerfaces 2007; 59(1): 105-11.

[15] Alvares DVD, de Andrade VAC, Mocelin AJ, Barbosa DS, Mise RA, Malsuo T. Folic acid therapy reduces plasma homocysteine levels and improvesplasma antioxidant capacity in hemodialysis patients. Nutrition 2007; 23(3): 242-7.

[16] Zhu A, Chen T. Blood compatibility of surface-engineered poly(ethylene terephthalate) viao-carboxymethylchitosan. Colloids Surf B: Biointerfaces 2006; 50(2): 120-5.

[17] Bing-lin $\mathrm{H}$, Wen-qiang $\mathrm{H}$. Ion exchange and adsorbing resin. Shanghai, Publishing company of Shanghai Technology and Education 1995.

[18] Lu L, Yuan Z, Shi K, et al. Study on the adsorption of Polycontaining amino-hydroxy ethyl methacrylate resin for bilirubin. China Univ Chem Acta 2003; 24: 454-8.

[19] Wu G, Shen Y, Xie A, Hongyun L. Study on the adsorption properties of Chitosan for bilirubin. Anhui Univ Acta 2003; 27: 8992.

[20] Wei B, Yuan Z, He B. Study on absorbent for bilirubin in hemoperfusion. Polym Bull 1997; 6:102-7.

[21] Willia RC, Richard JH, Michael JL. Effect of membrane composition and structure on solute removal and biocompatibility in hemodialysis. Kidney Int 1999; 56(6): 2005-2015.

[22] Lin W-C, Yu D-G, Yang M-C. Blood compatibility of thermoplastic polyurethane membrane immobi-lized with watersoluble chitosan/dextran sulfate. Colloids Surf B: Biointerfaces 2005; 8: 82-92.

[23] Reiser DJ. Neonatal jaundice: physiologic variation or pathologic process. Crit Care Nurs Clin North Am 2004; 16(2): 257-269.

[24] Ai Y, Bing J-H, Mao L. The clinical analysis of 112 pathological jaundice of newborn. J China Aristogenesis Heredity 2001; 9: 92-6. 